\title{
Aleukaemic leukostasis in a case of large cell non-Hodgkin's lymphoma: report of a case with a distinctive central nervous system involvement
}

\author{
S JAIN, D KOTASEK, PC BLUMBERGS, RE SAGE
}

From the Departments of Pathology and Clinical Haematology/Oncology, The Queen Elizabeth Hospital, Woodville, South Australia and the Department of Tissue Pathology, Institute of Medical and Veterinary Science, Adelaide, South Australia

SUMMARY A 52-year-old man with large cell non-Hodgkin's lymphoma developed a unique pattern of central nervous system involvement by lymphoma while in apparent systemic remission. Despite intermittent neurological episodes suggestive of CNS involvement by lymphoma no evidence of CNS lymphoma was found on repeated cerebrospinal fluid and brain CT scan examinations. At necropsy widespread occlusion of cerebral blood vessels by malignant lymphoid cells was observed with extensive tissue necrosis and haemorrhages. Leukaemic involvement of peripheral blood was not detected on repeated blood film examinations during life.

Involvement of the central nervous system (CNS) by non-Hodgkin's lymphoma is a well recognised complication of this disease. CNS involvement has been reported in 0 to $29 \%$ of patients with non-Hodgkin's lymphoma ${ }^{1-7}$ with an average frequency of $8.4 \%$ in a recent series. ${ }^{7}$ The highest incidence of CNS lymphoma is seen in patients with lymphoblastic lymphoma, diffuse undifferentiated lymphoma and Burkitt's lymphoma. ${ }^{136-8}$

Intravascular leukostasis resulting in the occlusion of small blood vessels, tissue ischaemia and necrosis is a recognised complication of the acute and chronic leukaemias in which the leukocyte count rises above 50-100000 leukocytes $/ \mathrm{mm}^{3} .^{9-11}$ Similar leukocyte count elevations have only rarely been reported in non-Hodgkin's lymphoma. Fram et al ${ }^{12}$ and Come et $a l^{13}$ did not report any cases of intravascular leukostasis in their series of non-Hodgkin's lymphoma patients in the leukaemic phase and no cases were reported in non-Hodgkin's lymphoma by Graus $e t$ $a l^{14}$ in a review of cerebrovascular complications in patients with cancer.

Address for reprint requests: Dr S Jain, Department of Pathology, The Queen Elizabeth Hospital, Woodville Road, Woodville, South Australia 5011.

Received 17 September 1985 and in revised form 11 December 1985. Accepted 15 December 1985
We report a case of diffuse histiocytic (large cell) non-Hodgkin's lymphoma in which diffuse intracerebral haemorrhages secondary to intravascular leukostasis by malignant lymphocytes developed during apparent systemic remission. Despite widespread tissue leukostasis, seen particularly in the cerebral vessels, peripheral blood leukocytosis was absent throughout the entire disease course.

\section{Case report}

A 52-year-old man presented in July 1982 with a one month's history of lassitude, sweats and left upper quadrant abdominal pain. Clinical examination showed fever and hepatosplenomegaly. A comprited tomography (CT) scan of the abdomen revealed a markedly enlarged liver and spleen but no lymphadenopathy. The abnormal laboratory investigations were: serum calcium $3.03 \mathrm{mmol} / \mathrm{l}(\mathrm{N}=$ 2.1-2.6), alkaline phosphatase (ALP) $483 \mathrm{U} / 1(\mathrm{~N}=35-100)$, aspartate transaminase (AST) $83 \mathrm{U} / 1(\mathrm{~N}=0-45)$, gamma glutamyl transpeptidase (GGT) $517 \mathrm{U} / \mathrm{l}(\mathrm{N}=0-70)$, lactate dehydrogenase (LD) $2040 \mathrm{U} / \mathrm{l}(\mathrm{N}=120-250), \mathrm{Hb} 17 \cdot 1 \mathrm{~g} / \mathrm{dl}$ $(\mathrm{N}=11 \cdot 5-16)$ and ESR $61 \mathrm{~mm}(\mathrm{~N}=0-7 \mathrm{~mm})$ in the first hour. The white cell count was $6.4 \times 10^{9} / 1$ with neutrophils $74 \%$, lymphocytes $16 \%$, monocytes $9 \%$ and basophil $1 \%$. Needle biopsy of the liver and posterior iliac crest bone marrow biopsies showed involvement with diffuse histiocytic lymphoma (Rappaport classification) ${ }^{16}$ or large cell lymphoma of immunoblastic type according to the NCI Work- 
ing Formulation classification. ${ }^{17}$ Prominent portal and intrasinusoidal infiltration by large, pleomorphic lymphoid cells was seen in the liver. Surface marker analysis of blood and bone marrow specimens was unsuccessful. The patient was staged as Stage IVB according to the Ann Arbor Conference criteria. ${ }^{18}$

Chemotherapy was begun using CHOP (cyclophosphamide, doxorubicin, vincristine and prednisolone) with rapid improvement. The patient remained well during the next four months although hepatomegaly and mild abnormalities of liver function tests persisted. In November 1982, the patient underwent an emergency laparotomy and splenectomy for a spontaneously ruptured spleen. Histological examination of the spleen (fig 1) and a liver biopsy specimen showed involvement with diffuse large cell lymphoma with prominent splenic intravascular and hepatic intrasinusoidal lymphoma cell infiltration. Immunohistochemical stains of the spleen indicated a null cell pattern.

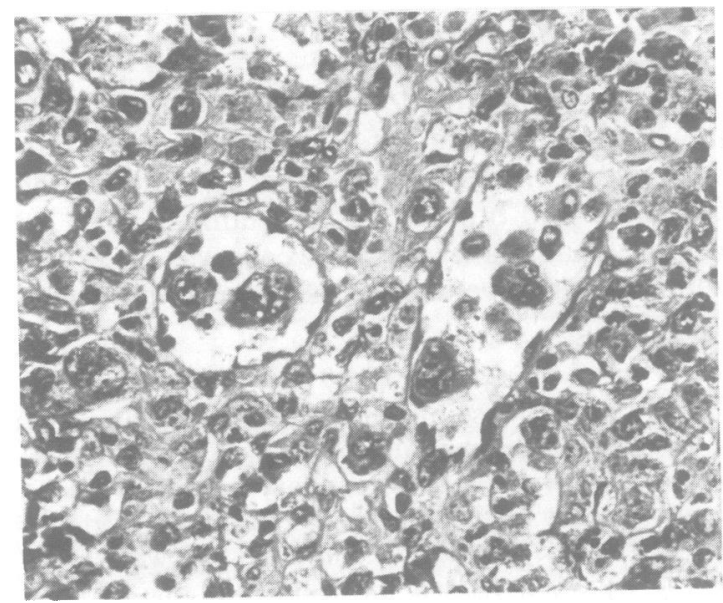

Fig 1 Splenic section. A diffuse infiltrate of large,

pleomorphic lymphoid cells with prominent nucleoli with

luminal involvement of numerous small blood vessels $(H \& E$, $\times 300$ ).

Chemotherapy was changed to C-MOPP (cyclophosphamide, vincristine, prednisolone and procarbazine). In March 1983 he presented with acute onset of diplopia and ataxia. Clinical examination showed horizontal nystagmus and cerebellar ataxia. The possibility of CNS involvement by non-Hodgkin's lymphoma was raised despite a normal CT brain scan and a normal lumbar puncture. Methotrexate $10 \mathrm{mg}$ was instilled intrathecally and CMOPP chemotherapy continued. The patient's symptoms resolved rapidly. A posterior iliac crest marrow biopsy at this time showed no evidence of lymphoma. C-MOPP was continued and the patient remained well until May 1983 when he presented with an influenza-like illness associated with high fever and meningism. No abnormalities were found on neurological examination. The CSF examination was normal and the patient recovered following symptomatic treatment. Chemotherapy with C-MOPP was continued.
Clinical remission continued until August 1983 three weeks after the last course of C-MOPP. The patient was admitted following a generalised seizure preceded by a 2 week history of intermittent speech and writing difficulties, confusion and memory lapses. Examination showed a confused, disoriented man with signs suggestive of bilateral cerebral hemisphere dysfunction including bilateral corticospinal tract involvement. CT brain scan was normal. Laboratory investigations were as follows: $\mathrm{Hb} 10.6 \mathrm{~g} / \mathrm{dl}$, WCC $12.3 \times 10^{9} / 1$ (neutrophils $64 \%$, lymphocytes $25 \%$, monocytes $9 \%$ ), platelets $325 \times 10^{9} / 1$, Calcium $2.39 \mathrm{mmol} / 1(\mathrm{~N}=$ 2.1-2.6), ALP $198 \mathrm{U} / 1(\mathrm{~N}=35-100)$, GGT $124 \mathrm{U} / 1(\mathrm{~N}=$ $0-70)$ and LD $777 U / 1(N=120-250)$. The only abnormality on CSF examination (including cytology) was a CSF protein level of $1.03 \mathrm{~g} / 1(\mathrm{~N}=0 \cdot 16-0.50 \mathrm{~g} / \mathrm{l})$. Intravenous dexamethasone and phenytoin were given without improvement. An electroencephalogram was interpreted as consistent with a diffuse encephalopathic process which affected the left cerebral hemisphere to a greater extent. A repeat CT brain scan two days later disclosed a hypolucent area in the left parieto-occipital lobe. Plans for a burr-hole biopsy of this lesion were abandoned when the patient's condition rapidly deteriorated. Repeat CSF examination at this time showed no abnormal lymphoid cells, a CSF protein of $1.28 \mathrm{~g} / 1(\mathrm{~N}=$ $0.16-0.50 \mathrm{~g} / \mathrm{l})$ and $12.5 \mathrm{mg}$ of methotrexate was instilled. Within 18 hours the patient's clinical state improved dramatically with a substantial resolution of the previously abnormal neurological findings.

A further CT brain scan performed 5 days after admission disclosed an additional hypolucent area within the body of the right caudate nucleus. On day 7 the patient's condition deteriorated rapidly with the development of bilateral pyramidal signs, loss of speech and coma. A further injection of $12.5 \mathrm{mg}$ of intrathecal methotrexate was given. CT brain scan on day 9 showed an intracerebral haemorrhage in the right internal capsule in addition to the previous findings. The patient died thirteen days after admission.

\section{Necropsy findings}

The brain contained multiple foci of recent haemorrhage varying in size from $1-2 \mathrm{~mm}$ to $30 \mathrm{~mm}$ in diameter involving the central gray and white matter, subcortical white matter and cerebral cortex of both hemispheres. Similar lesions were present in the brainstem and cerebellum. The haemorrhagic lesions were the result of coalescent petechial haemorrhages (fig 2) the largest lesion affecting the right basal ganglia and internal capsule. Microscopically the haemorrhages were all related to intravascular leukostasis in small arteries, arterioles, capillaries and venules by large numbers of abnormal lymphoid cells (fig 3). Many blood vessels showed angionecrosis with fibrinoid degeneration of the vessel walls and extravasation of red blood cells into the surrounding neuropil (fig 4). Invasion of vessel walls or perivascular spaces by lymphoid cells was not seen. Multiple small areas of ischaemic necrosis of varying ages were also seen throughout the CNS. Meningeal or direct parenchymal involvement by lymphoma was not present and all large intracranial and extracranial vessels, cranial nerves and the dura mater were normal. Focal lymphoma deposits were seen in the liver and both kidneys. The right renal lymph node group was completely replaced by lymphoma while all 


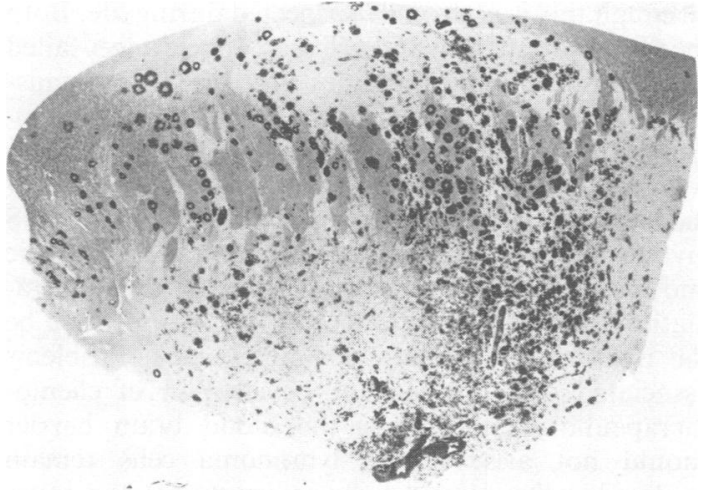

Fig 2 Right caudate nucleus, internal capsule and putamen showing coalescent petechial haemorrhages many with central leukostasis (Weil stain, $\times 2$ ).

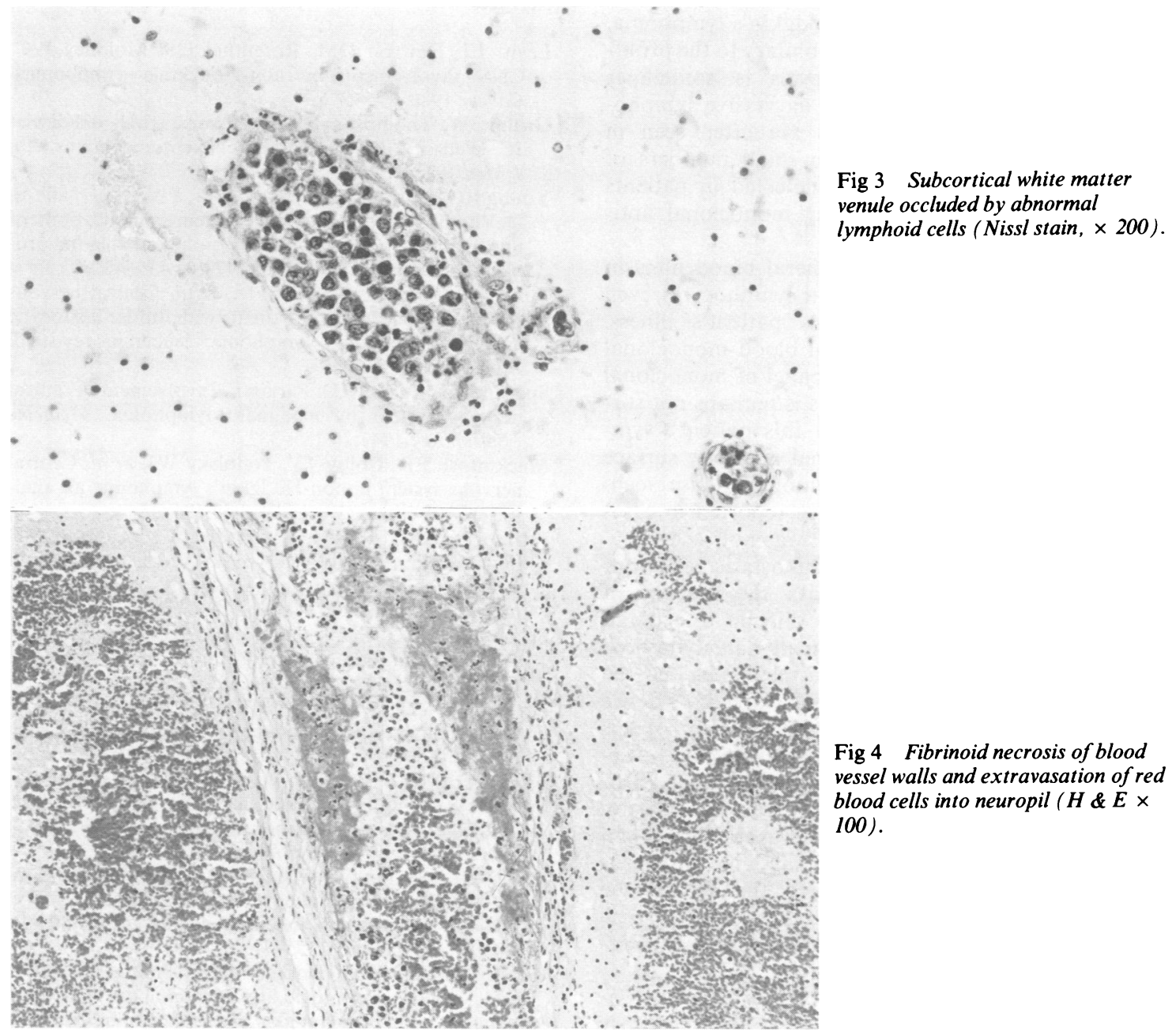

other lymph nodes and the bone marrow were free of lymphoma. The heart, lungs, pituitary and thyroid glands contained intravascular aggregates of large, atypical pleomorphic lymphoid cells which were morphologically similar to the cells in the bone marrow and liver biopsies at presentation and to the cells seen in the CNS, liver and spleen at autopsy. An occasional blood vessel was completely occluded by lymphoid cells, but there was no evidence of necrosis, thrombosis or haemorrhage similar to that seen in the CNS.

\section{Discussion}

Involvement of the CNS by malignant lymphoma is a well recognised complication of this disease. The non-Hodgkin's lymphoma is 0 to $29 \%$ in several series. $^{1-8}$ Leptomeningeal and focal parenchymal unle occluded by abnormal $\times 200)$

Fig 4 Fibrinoid necrosis of blood blood cells into neuropil ( $H \& E \times$ 100). reported range of incidence of CNS involvement by 
lymphomatous involvement are the most common forms of CNS disease seen at necropsy. Other modes of CNS involvement seen less frequently are extradural or bone involvement extending into the CNS and cranial or spinal nerve infiltration. ${ }^{168}$ We report an additional unique pattern of CNS damage secondary to widespread intravascular leukostasis by malignant lymphoma cells in a patient with diffuse histiocytic non-Hodgkin's lymphoma. At necropsy, extensive CNS ischaemic and haemorrhagic lesions directly responsible for the patient's death were found. These lesions resulted from the leukostatic occlusion by malignant lymphoid cells of small and medium sized blood vessels of the brain. None of the usual patterns of CNS lymphoma was present.

While intravascular leukostasis is a well recognised phenomenon in the acute and chronic leukaemias $^{9-11}$ this complication is rarely if ever observed in patients with non-Hodgkin's lymphoma. Peripheral blood leukocytosis secondary to the proliferation of malignant lymphocytes is sometimes observed in advanced stages of aggressive lymphomas. ${ }^{1213}$ Atypical lymphocytes are often seen in peripheral blood films and even small numbers of malignant lymphocytes can be detected in patients with malignant lymphoma using monoclonal antibodies and flow cytometry. ${ }^{15}$

A careful review of the peripheral blood films in this case failed to reveal atypical lymphocytes even during the final stages of this patient's illness. Repeated search for a peripheral blood monoclonal lymphocyte population using a panel of monoclonal antibodies was unsuccessful. This is perhaps not surprising in view of the fact that this patient's lymphoma was "null" by monoclonal antibody surface marker analysis and the immunoperoxidase technique. Leukocytosis of the degree reported in cases with intravascular leukostasis ${ }^{910}$ was not seen.

The unusual angiophilic and leukostatic behaviour of this lymphoma, together with the absence of peripheral blood involvement, is difficult to explain. Changes on the surface of the malignant lymphoid cells resulting in affinity between these cells and the endothelium can be proposed but we lack evidence for this hypothesis. A second puzzling feature of this case was the confinement of the severe and widespread leukostatic tissue damage to the CNS. Although a minor degree of blood vessel occlusion was seen in other organs, the CNS was the only organ where tissue ischaemia, necrosis and haemorrhages were present. Whether this relates to the fact that the cerebral vessels affected were end arteries or to some other property of the cerebral vasculature is uncertain.

The diagnosis of this CNS complication of nonHodgkin's lymphoma was not made until necropsy although this was strongly suspected during life. Both the CSF examination and CT scan appearances failed to disclose any abnormalities until the final admission. Treatment with systemic and intrathecal chemotherapy resulted in clinical improvement in the neurological symptoms on several occasions. Currently the treatment of non-Hodgkin's lymphoma with CNS involvement utilises single and combination systemic and intrathecal chemotherapy as well as cranial irradiation. Systemic chemotherapy would appear to be the treatment of choice in similar cases. Problems associated with the lack of penetration of chemotherapeutic agents across the blood brain barrier should not arise if the lymphoma cells remain confined to the intravascular compartment and cause derangements by virtue of vascular occlusion.

\section{References}

${ }^{1}$ Levitt LJ, Dawson DM, Rosenthal DS, Moloney WC. CNS involvement in non-Hodgkin's lymphomas. Cancer 1980;45:545-52.

${ }^{2}$ Griffin JW, Thompson RW, Mitchinson MJ, deKiewiet JC, Welland FH. Lymphomatous leptomeningitis. Am J Med 1971;51:200-8.

${ }^{3}$ Young RC, Howser DM, Anderson T, Fisher RI, Jaffe E, DeVita VT. Central nervous system complications of non-Hodgkin's lymphoma. The potential role for prophylactic therapy.Am J Med 1979;66:435-43.

${ }^{4}$ Bunn PA, Schein PS, Banks PM, et al. Central nervous system complications in patients with diffuse histiocytic and undifferentiated lymphoma: leukemia revisited. Blood 1976;47:3.

${ }^{5}$ Sparling HJ, Adams RD, Parker F. Involvement of central nervous system by malignant lymphoma. Medicine (Baltimore) 1947;26:285-332.

${ }^{6}$ Mackintosh FR, Colby TV, Podolsky WJ, et al. Central nervous system in non-Hodgkin's lymphoma: an analysis of 105 cases. Cancer 1982;49:586-95.

7 Johnson GJ, Oken MM, Anderson JR, O'Connell MJ, Glick JH. Central nervous system relapse in unfavourable-histology non-Hodgkin's lymphoma: is prophylaxis indicated? Lancet 1984;ii:685-7.

${ }^{8}$ Schaumburg HH, Plank CR, Adams RD. The reticulum cell sarcoma-microglioma group of brain tumours. Brain 1972;95:119-212.

${ }^{9}$ Freireich EJ, Thomas LB, Frei E, Fritz RD, Forkner CE. A distinctive type of intracerebral hemorrhage associated with "blastic crisis" in patients with leukemia. Cancer 1960;13:146-54.

${ }^{10}$ McKee LC, Collins RD. Intravascular leukocyte thrombi and aggregates as a cause of mobidity and mortality in leukemia. Medicine (Baltimore) 1974;53:463-78.

${ }^{11}$ Thomas LB. Pathology of leukemia in the brain and meninges: postmortem studies of patients with acute leukemia and of mice given inoculations of L1210 leukemia. Cancer Res 1965;25:1555-71.

${ }^{12}$ Fram RJ, Skarin AJ, Rosenthal DS, Pinkus G, Nadler LM. Clinical, pathologic and immunologic features of patients with non-Hodgkin's lymphoma in leukemic 
phase. Cancer 1983;52:1220-8.

${ }^{13}$ Come SE, Jaffe ES, Anderson JC, et al. Non-Hodgkin's lymphomas in leukemic phase: Clinicopathological correlations. Am J Med 1980;69:667-74.

${ }^{14}$ Graus F, Rogers LR, Posner JB. Cerebrovascular complications in patients with cancer. Medicine (Baltimore) 1984;64(1):16-35.

${ }^{15}$ Smith BR, Weinberg DS, Robert NJ, et al. Circulating monoclonal B lymphocytes in non-Hodgkin's lymphoma. N Engl J Med 1984;311:1476-81.
${ }^{16}$ Rappaport $H$. Tumors of the hemopoietic system. In: Atlas of Tumor Pathology, Sec. III, Fascicle 8. Washington, DC. Armed Forces Institute of Pathology 1966.

${ }^{17}$ National Cancer Institute sponsored study of classifications of non-Hodgkin's lymphomas. Summary and description of a working formulation for clinical usage. Cancer 1982;49:2112-35.

${ }^{18}$ Carbone PP, Kaplan HS, Musshoff K, et al. Report of the Committee on Hodgkin's disease staging. Cancer Res 1971;31:1860-1. 